\title{
Line-Of-Sight Interference Statistics and User Scheduling with 3D Angular Separation
}

\author{
Yawei Yu $\left(\mathbb{D}\right.$, Jianhua Zhang ${ }^{D}$, and Lei Tian \\ Beijing University of Posts and Telecommunications, Beijing 100876, China \\ Correspondence should be addressed to Jianhua Zhang; jhzhang@bupt.edu.cn
}

Received 27 November 2017; Accepted 3 June 2018; Published 8 July 2018

Academic Editor: Shiwen Yang

Copyright (@) 2018 Yawei Yu et al. This is an open access article distributed under the Creative Commons Attribution License, which permits unrestricted use, distribution, and reproduction in any medium, provided the original work is properly cited.

\begin{abstract}
We focus on the interference statistical metrics of matched filter transmission over line-of-sight (LoS) downlink channels. The exact moments of the interference are derived, which can be used to make approximations for user rates and gain insights into the effects of system parameters such as angle spread (AS), antenna numbers, and array spacings. As the interference decreases with larger AS, we propose a user scheduling scheme by grouping users into different subspaces with corresponding orthogonal fraction of resource blocks, aiming to maximize the 3D angular separation among multiusers within each group; thus, a lower interference and higher sum rate would be expected. By performing this user scheduling scheme with $3 \mathrm{D}$ angular separation, a large sum rate gain is observed, which provides us a promising method in interference control and management.
\end{abstract}

\section{Introduction}

The growing importance of massive MIMO [1], small cell [2], and millimeter wave [3] communications is motivating an increased interest in line-of-sight (LoS) channels [4] and simple transmission schemes such as matched filter (MF) precoding [5]. However, interference will arise in such multiuser systems with MF transmission [6], which will degrade the system performance [7], thus drawing the researchers' attention [8].

Considering a single-cell massive MIMO system where $K$ single-antenna users are evenly distributed around the base station (BS) in the horizontal plane, the favorable propagation conditions of LoS channels, which are defined as mutual orthogonality among the vector-valued channels to the terminals with no mutual interference, are well investigated in [9]. As the mutual orthogonality among LoS channels is closely related to the antenna array at BS, the impact of different antenna parameters on asymptotic orthogonality, including the array geometries [10], antenna spacings [11], and antenna numbers [12], was studied. Using a uniform rectangular array (URA) at BS for the LoS MIMO system, results in [13] show that the ergodic rate is maximized for antenna spacing equal to integer multiples of one-half wavelength while the achievable sum rate with other antenna arrays can be found in [14]. In LoS channels, the phase of the received signal is determined by the relative location of the user (to BS); the work in [15] demonstrates that the user interference is partially determined by the LoS angle of arrival of signals. The deterministic dependence of interference on locations poses a significant challenge in modelling the interference distribution; the authors in [16] show that the main lobe distribution of LoS interference can be approximated by a beta mixture in the uplink LoS channel. As interference distribution can be used to compute signalto-noise-plus-interference ratio (SINR) metrics such as outage probability and average throughput, prior work on LoS interference modeling is summarized in [17] and the interference model based on SINR is proposed and emphasized. The authors in [18] propose a statistical-eigenmode spacedivision multiple-access downlink transmission scheme based on the ergodic SINR and present the achievable sum rate of the multiuser system. This work also studies, for a different reason, the interference variables considered here.

As the sum rate of the multiuser MIMO system with interference is closely related to the SINR at each user, optimizing the SINR is key to improve the system performance [19]. Framework on transceiver (precoder and decoder) 
designing for interference alignment (IA) is presented [20], and different IA schemes based on resource allocation in multiuser MIMO systems are summarized [21]. By grouping users with different mobilities to have different coherence times, the interference signal only occupies limited dimensions and the degrees of freedom for the LoS interference channel are improved due to this blind IA scheme [22]. Furthermore, IA via a time-indexed interference graph for LoS channels is studied in [23], a maximal independent set is presented via dynamic programing, and orthogonal interference subspaces are designed to combat the interference at each user. Considering the MIMO downlink channel in a multiuser system, an efficient suboptimal algorithm of user scheduling is given by assigning the coordinates of transmission space to different users, aiming to achieve the best performance in terms of the sum rate throughput [24]. By allocating various resource blocks (e.g., time, frequency, and power) to users in different groups, the multiuser interference would remain within acceptable limits [25].

As we can summarize, all of the above work is closely related to the statistic characteristics of LoS interference; however, the derivation of a multiuser interference is difficult due to integrations of complicated components such as different elevation angle distributions and exponential antenna radiation patterns; thus, work on exact expressions of the interference moments is rare. Furthermore, conventional work all assumes that multiusers are evenly or randomly distributed around BS in the azimuth domain with no consideration of the elevation counterpart, which is not that realistic especially for 3D propagation scenarios with multiusers in high-rise buildings. For a 3D LoS propagation channel, the interference model is lacking and the effects of system parameters including the angle spread (AS), antenna spacing, and antenna numbers on the interference still remain unknown. Till now, there are various IA schemes such as resource allocations in the time, frequency, and power domains; the utilization of the degree of freedom in 3D space is rarely reported. The overall performance of multiuser MIMO systems is a complex joint multiobjective optimization problem since many variables and parameters have to be optimized, including the selection of users, the number and interval spacing of antennas, power allocation, and transmission technique. Based on the prior work, we make the following contributions:

(1) Considering the LoS downlink channel with a simple MF transmission scheme and multiusers are distributed in the 3D space, we derive the exact expressions of the interference moments and present the simulative interference results under different antenna numbers, antenna spacings, and AS values. As usual, the multiusers are assumed as evenly distributed around the BS in the azimuth domain and von Mises (VM) distribution in the elevation counterpart [26].

(2) Comparative results of the interference against AS, antenna numbers, and antenna spacings demonstrate that the averaged interference decreases rapidly when the elevation angle spread (EAS) increases

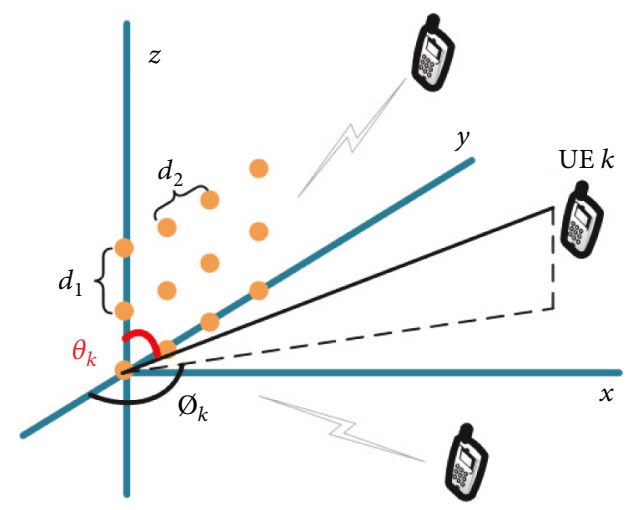

FIgURE 1: Multiuser LoS interference with URA at BS.

(or the antenna number decreases, antenna spacing increases), which enables us to reduce the interference once users are scheduled to maintain decent angular separations (or finite antenna activation, antenna configuration optimization). While the mean interference level falls with increasing AS, there remains large variability (see the results of interference variance), especially in the super dense area with $\mathrm{EAS}<10^{\circ}$, which poses a severe challenge to simple MF processing.

(3) Except IA schemes achieved by user scheduling in time, frequency, and power domains as mentioned above, we present the user scheduling via $3 \mathrm{D}$ space utilization as well. On the criteria of maximizing the angular separations among users within each group, multiusers are divided into different groups and occupy corresponding orthogonal fraction of wireless resource blocks. By performing the user scheduling scheme via $3 \mathrm{D}$ angular separation, a large sum rate gain is obtained and will be further enhanced by larger EAS and antenna spacing.

(4) We conclude that by performing the user scheduling scheme via 3D angular separation, a significant system sum rate gain can be achieved, which ensures the promising future of $3 \mathrm{D}$ space utilization in interference management.

The remainder of this article is outlined as follows: in Section 2, we give the system model and performance metrics. In Sections 3 and 4, we derive the interference moments and present the results of interference against AS and antenna spacing. In Section 5, the user scheduling scheme is put forwarded along with the simulative results of the system sum rate. Then, conclusions are drawn in Section 6.

\section{System Model and Performance Metrics}

Given the typical URA at BS, as shown in Figure 1, there are $M$ vertical elements spaced by $d_{1}$ wavelength and $N$ horizontal elements with $d_{2}$ wavelength spacing. For simplicity, we assume $K$ single-antenna users (UEs) being served in a pure LoS environment within a single cell and the LoS angle of UE 
$k$ is denoted by $\left(\varphi_{k}, \theta_{k}\right)$. The channel impulse response (CIR) between UE $k$ and the URA, $\mathbf{H}_{k}$, is given by [27]

$$
\mathbf{H}_{k}=\sqrt{\beta_{k}} \mathbf{H}_{h, k} \otimes \mathbf{H}_{v, k}
$$

where

$$
\begin{aligned}
\mathbf{H}_{v, k} & =\left[1, e^{-j u_{k}}, e^{-j 2 u_{k}}, \ldots, e^{-j(M-1) u_{k}}\right]^{T}, \\
\mathbf{H}_{h, k} & =\left[1, e^{-j v_{k}}, e^{-j 2 v_{k}}, \ldots, e^{-j(N-1) v_{k}}\right], \\
u_{k} & =\frac{2 \pi}{\lambda} d_{1} \cos \theta_{k}, \\
v_{k} & =\frac{2 \pi}{\lambda} d_{2} \sin \theta_{k} \cos \phi_{k} .
\end{aligned}
$$

$\beta_{k}$ is the link gain, and $\lambda$ is the wavelength.

We next rewrite the $M \times N$ matrix $\mathbf{H}_{k}$ as a vector $\mathbf{h}_{k}$ in a dimension of $1 \times(M N)$ as follows:

$$
\begin{aligned}
& \mathbf{h}_{k}=\sqrt{\beta_{k}} \mathbf{a}_{k}, \\
& \mathbf{a}_{k}=\left[\mathbf{H}_{h, k}, e^{-j u_{k}} \mathbf{H}_{h, k}, e^{-j 2 u_{k}} \mathbf{H}_{h, k}, \ldots, e^{-j(M-1) u_{k}} \mathbf{H}_{h, k}\right] .
\end{aligned}
$$

Thus, the received downlink signal at UE $k$ is obtained

$$
y_{k}=\mathbf{h}_{k} \mathbf{x}+n_{k}
$$

where $\mathbf{x}$ is the transmitted signal and $n_{k} \sim \mathscr{C} \mathcal{N}\left(0, \sigma_{z}^{2}\right)$ is the additive white Gaussian noise with mean value 0 and variance $\sigma_{z}^{2}$.

Assuming linear precoding at the BS,

$$
\mathbf{x}=\mathbf{G d}
$$

where $\mathbf{G}=\left[\mathbf{g}_{1}, \mathbf{g}_{2}, \ldots, \mathbf{g}_{\mathbf{K}}\right]$ is a $M N \times K$ precoding matrix and $d=\left[d_{1}, d_{2}, \ldots, d_{K}\right]^{T}$ contains the transmitted data. If matched filter precoding is employed at the BS, then $g_{k}=$ $\mathrm{h}_{k}^{+}\left(M N \beta_{k}\right)^{-1 / 2}$, and the SINR at UE $k$, assuming $E\left[\left|d_{\kappa}\right|^{2}\right]=$ 1 , is given by

$$
\operatorname{SINR}_{k}=\frac{\left|\mathbf{h}_{k} \mathbf{g}_{k}\right|^{2}}{\sum_{j \neq k}^{K}\left|\mathbf{h}_{k} \mathbf{g}_{j}\right|^{2}+\sigma_{z}^{2}}=\frac{M N \beta_{k}}{\beta_{k} / M N \sum_{j \neq k}^{K}\left|\mathbf{a}_{k} \mathbf{a}_{j}^{+}\right|^{2}+\sigma_{z}^{2}}
$$

In (7), we observe that the intracell interference to UE $k$ from the transmitted signal to UE $j$ is $\beta_{k} I_{k j} /(M N)$, where

$$
I_{k j}=\left|\mathbf{a}_{k} \mathbf{a}_{j}^{+}\right|^{2} .
$$

Thus, the total intracell interference to UE $k$ is $\beta_{k} I_{k}$ l $(M N)$, where

$$
I_{k}=\sum_{j \neq k}^{K} I_{k j}
$$

The desired signal power is $M N \beta_{k}$; the SINR and the rate of UE $k$ can be expressed as

$$
\begin{aligned}
\operatorname{SINR}_{k} & =\frac{M N \beta_{k}}{\left(\beta_{k} / M N\right) I_{k}+\sigma_{z}^{2}}, \\
R_{k} & =\log _{2}\left(1+\frac{M N \beta_{k}}{\left(\beta_{k} / M N\right) I_{k}+\sigma_{z}^{2}}\right) .
\end{aligned}
$$

We note that the moments can be used to estimate the mean rate through the delta method [28], where the firstand second-order center moments of $I_{k}$ are included:

$$
\mathbb{E}\left[R_{k}\right] \approx \log _{2}\left(1+\frac{1}{\mathbb{E}[\delta]}\right)+\gamma
$$

where

$$
\begin{aligned}
& \delta=\frac{1}{M N}\left(\frac{I_{k}}{N M}+\frac{\sigma^{2}}{\beta_{k}}\right), \\
& \gamma=\frac{\operatorname{Var}(\delta)}{2 \ln 2}\left[\frac{1}{\mathbb{E}(\delta)^{2}}-\frac{1}{(\mathbb{E}(\delta)+1)^{2}}\right] .
\end{aligned}
$$

Clearly, the performance is critically dependent on the distribution of the interference $I_{k}$. Hence, we will derive the moments of $I_{k}$, that is, $\mathbb{E}\left[I_{k}\right]$ and $\operatorname{Var}\left[I_{k}\right]$.

The other important variable governing the performance is the distribution of the LoS angles, $\left\{\left(\phi_{k}, \theta_{k}\right), k=1,2, \ldots\right.$, $K\}$. We assume the uniform distribution in azimuth domain with $\phi_{k} \sim U[-\pi, \pi]$ and the von Mises distribution in elevation domain with $\theta_{k} \sim \operatorname{VM}(\kappa, \mu)$, where the density function is

$$
f(\theta)=\frac{e^{\kappa \cos (\theta-\mu)}}{2 \pi I_{0}(\kappa)}, \quad 0 \leq \theta \leq \pi
$$

Here, $\kappa$ is a concentration parameter, $\mu$ is a location parameter, and $I_{0}(\cdot)$ is the modified Bessel function of the first kind in the zeroth order.

\section{Interference Moments}

From (11)-(13), we observe that $E\left[I_{k}\right]$ and $\operatorname{Var}\left(I_{k}\right)$ are required to compute the sum rate. Hence, we compute the first two moments of $I_{i}$ based on the following lemma.

Lemma 1. When $\phi \sim \mathcal{U}[-\pi, \pi]$ and $\theta \sim V M(\kappa, \pi / 2)$ with $f(\theta)$ in (14), the approximation

$$
e^{\kappa \cos (\theta-\pi / 2)} \approx\left(e^{\kappa}-1\right) \sin \theta^{L}+1,
$$

with the approximation accuracy validated in Figure 2, enables us to obtain

$$
\begin{aligned}
\mathbb{E}\left[e^{-j(m-p) u_{k}} e^{\left.-j(n-q) u_{k}\right] \triangleq}\right. & F(m-p, n-q, \kappa) \\
\approx & A+B+b J_{0}\left(-2 \pi d_{z}^{m, p}\right) C \\
& +2 b \sum_{r=1}^{R} j^{r} J_{r}\left(-2 \pi d_{z}^{m, p}\right) D
\end{aligned}
$$

where 


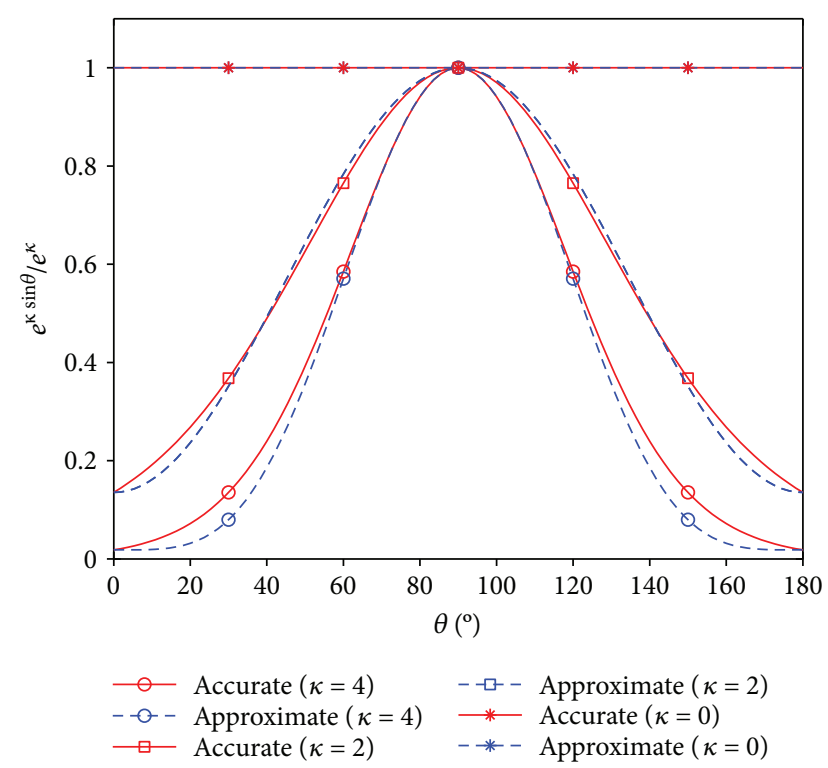

Figure 2: Approximation accuracy of $e^{\kappa \sin \theta} / e^{\kappa} \approx\left(\left(e^{\kappa}-1\right) \sin \right.$ $\left.\theta^{2 L}+1\right) / e^{\kappa}$.

$$
\begin{aligned}
A= & a \pi J_{0}\left(-2 \pi d_{z}^{m, p}\right) J_{0}^{2}\left(\pi d_{y}^{n, q}\right), \\
B= & 2 a \sum_{r=1}^{R} j^{r} J_{r}\left(-2 \pi d_{z}^{m, p}\right) \pi \cos \left(\frac{r}{2} \pi\right) J_{-r / 2} \\
& \cdot\left(\pi d_{y}^{n, q}\right) J_{r / 2}\left(\pi d_{y}^{n, q}\right),
\end{aligned}
$$

when $L=2 L_{0}$ is even in (15),

$$
\begin{aligned}
& C=\sum_{l=0}^{L_{0}} k_{0} \pi \cos (l \pi) J_{-l}\left(\pi d_{y}^{n, q}\right) J_{l}\left(\pi d_{y}^{n, q}\right) \\
& \begin{aligned}
D=\sum_{l=0}^{L_{0}} \frac{k_{l}}{2} \pi\left[\cos \left(l_{1} \pi\right) J_{-l_{1}}\left(\pi d_{y}^{n, q}\right) J_{l_{1}}\left(\pi d_{y}^{n, q}\right)\right. \\
\left.+\cos \left(l_{2} \pi\right) J_{-l_{2}}\left(\pi d_{y}^{n, q}\right) J_{l_{2}}\left(\pi d_{y}^{n, q}\right)\right] .
\end{aligned}
\end{aligned}
$$

when $L=2 L_{1}-1$ is odd in (15),

$$
\begin{gathered}
C=\sum_{l=1}^{L_{1}} k_{0} \pi \sin \left(\left(l-\frac{1}{2}\right) \pi\right) J_{-(l-(1 / 2))}\left(\pi d_{y}^{n, q}\right) J_{l-(1 / 2)}\left(\pi d_{y}^{n, q}\right) \\
D=\sum_{l=1}^{L_{1}} \frac{k_{l}}{2} \pi\left[\sin \left(l_{3} \pi\right) J_{-l_{3}}\left(\pi d_{y}^{n, q}\right) J_{l_{3}}\left(\pi d_{y}^{n, q}\right)\right. \\
\left.+\sin \left(l_{4} \pi\right) J_{-l_{4}}\left(\pi d_{y}^{n, q}\right) J_{l_{4}}\left(\pi d_{y}^{n, q}\right)\right]
\end{gathered}
$$

The parameters mentioned in (16)-(22) are given below:

(i) $d_{y}^{n, q}$ and $d_{z}^{m, p}$ are defined as

$$
\begin{aligned}
d_{y}^{n, q} & =\frac{(n-q) d_{2}}{\lambda}, \\
d_{z}^{m, p} & =\frac{(m-p) d_{1}}{\lambda} .
\end{aligned}
$$

(ii) $a$ and $b$ are defined as

$$
\begin{aligned}
& a=\frac{1}{2 \pi I_{0}(\kappa)}, \\
& b=\frac{e^{k}-1}{2 \pi I_{0}(\kappa)} .
\end{aligned}
$$

(iii) $l_{1}, l_{2}, l_{3}$, and $l_{4}$ are defined as

$$
\begin{aligned}
& l_{1}=\frac{2 l+r}{2}, \\
& l_{2}=\frac{2 l-r}{2}, \\
& l_{3}=\frac{2 l-1+r}{2}, \\
& l_{4}=\frac{2 l-1-r}{2} .
\end{aligned}
$$

(iv) $k_{l}$ are coefficients of $\sin \theta^{L}$ expansion in [29, eq. (1.320.1)]:

when $L=2 L_{0}$ is even,

$$
\begin{aligned}
\sin \theta^{L} & =\sum_{l=0}^{L_{0}} k_{l} \cos 2 l \theta, \\
k_{0} & =\frac{1}{2^{2 L_{0}}}\left(\begin{array}{c}
L_{0} \\
2 L_{0}
\end{array}\right) \\
k_{l} & =\frac{(-1)^{l}}{2^{2 L_{0}-1}}\left(\begin{array}{c}
L_{0}-l \\
2 L_{0}
\end{array}\right), \\
L & =1,2, \ldots L_{0} .
\end{aligned}
$$

when $L=2 L_{1}-1$ is odd,

$$
\begin{aligned}
\sin \theta^{L} & =\sum_{l=1}^{L_{1}} k_{l} \sin ((2 l-1) \theta), \\
k_{l} & =\frac{(-1)^{l-1}}{2^{2 L_{1}-2}}\left(\begin{array}{c}
L_{1}-l \\
2 L_{1}-1
\end{array}\right), \quad L=1,2, \ldots, L_{1} .
\end{aligned}
$$

Proof 1. See Appendix A.

Since the double integral $\mathbb{E}\left[e^{-j(m-p) u_{k}} e^{-j(n-q) v_{k}}\right]$ occurs throughout the moment derivations, we use the short notation, $F(m-p, n-q, \kappa)$, defined in (16). From Lemma 1 , the following results can be obtained. 


\section{Theorem 1.}

$$
\mathbb{E}\left[I_{k}\right]=(K-1) \sum_{m, n, p, q}|F(m-p, n-q, \kappa)|^{2},
$$

where $\sum_{m, n, p, q}$ denotes $\sum_{m=0}^{M-1} \sum_{n=0}^{N-1} \sum_{p=0}^{M-1} \sum_{q=0}^{N-1}$.

Proof 2. See Appendix B.

\section{Theorem 2.}

$$
\begin{aligned}
\mathbb{E}\left[I_{k}^{2}\right]= & (K-1)^{2} \sum_{p_{1}} \sum_{q_{1} m_{1} n_{1} p_{2} q_{2} m_{2} n_{2}} \\
& \cdot F\left(m_{1}+m_{2}-p_{1}-p_{2}, n_{1}+n_{2}-q_{1}-q_{2}-q_{2}, \kappa\right) \\
& \cdot F\left(-\left(m_{1}-p_{1}\right),-\left(n_{1}-q_{1}\right), \kappa\right) \\
& \cdot F\left(-\left(m_{2}-p_{2}\right),-\left(n_{2}-q_{2}\right), \kappa\right) .
\end{aligned}
$$

Proof 3. See Appendix C.

From Theorems 1 and 2, the variance of $I_{k}$ follows from

$$
\operatorname{Var}\left[I_{k}\right]=\mathbb{E}\left[I_{k}^{2}\right]-\left(\mathbb{E}\left[I_{k}\right]\right)^{2}
$$

Note that, when $\kappa=0$, we have the special case where elevation angle is uniformly distributed with $\theta \sim U[0, \pi]$.

\section{Simulative Results of Interference and Analysis}

4.1. Numerical and Simulation Results: Moments. The interference is closely related to the multiuser angle distribution in both elevation and azimuth domains. By following (16) in Lemma 1 and Theorem 1, we present the interference variation against elevation angle spread under various antenna spacings in Figure 3 when considering an even distribution of azimuth angle over $[0,2 \pi]$.

Specifically, results are shown for the rectangular antenna array with $100(10 \times 10)$ and $36(6 \times 6)$ elements at BS with antenna spacing $d=0.25 \lambda, d=0.5 \lambda$, and $d=1 \lambda\left(d_{1}=d_{2}=d\right)$ when $K=5$. For all cases, the simulative results of the approximate exact moments fit quite well with the true value; thus, the derivation of Lemma 1 based on the simple approximation in (15) provides us a general method to calculate the mean interference with high approximation accuracy. Obvious features of the results include a drop in the mean interference as EAS increases ( $\kappa$ decreases), as antenna spacing increases, and as antenna number at BS decreases. Furthermore, we note that for small EASs, the interference is massive and stays stable, which should account for the tiny variation of the term $e^{-j(m-p) u_{k}} e^{-j(n-q) v_{k}}$ in (16) for small EAS $\left(<10^{\circ}\right)$ in the super dense area. When the EAS increases from $10^{\circ}$ to $40^{\circ}$, defined as dense area, and the mean interference $\mathbb{E}\left|I_{k}\right|$ decreases rapidly, then it reaches the bottom gradually with a long flat tail in the sparse area with large EASs $\left(40^{\circ} \sim 90^{\circ}\right)$. Moreover, a value of $(M N)^{2}$ makes the interference power equal to the signal power, and this is exceeded by many of the scenarios. Clearly, either low

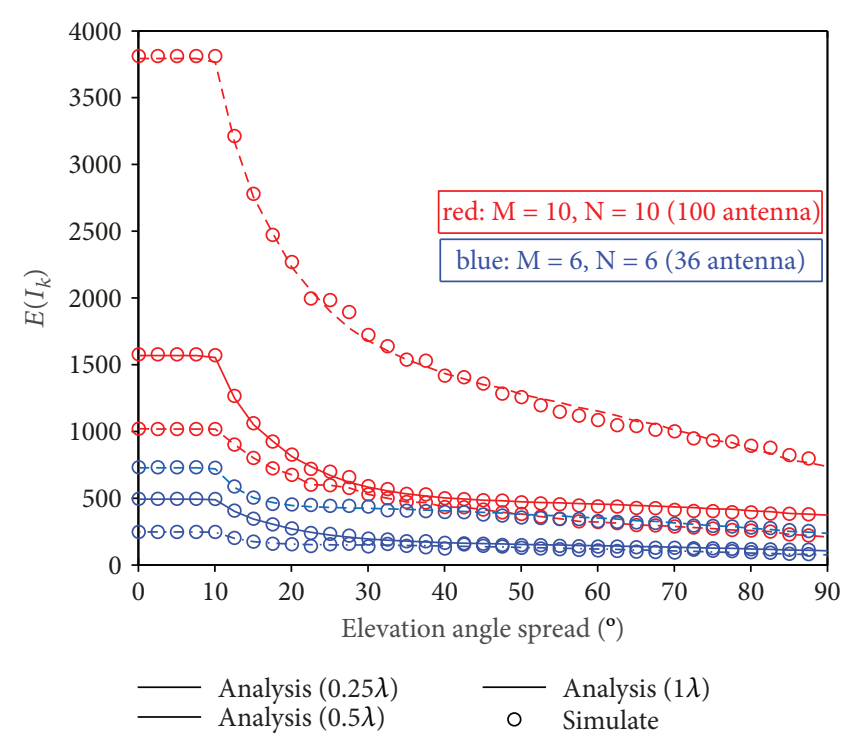

FIgURE 3: Mean interference $E\left[I_{k}\right]$ against elevation angle spread: $K=5$.

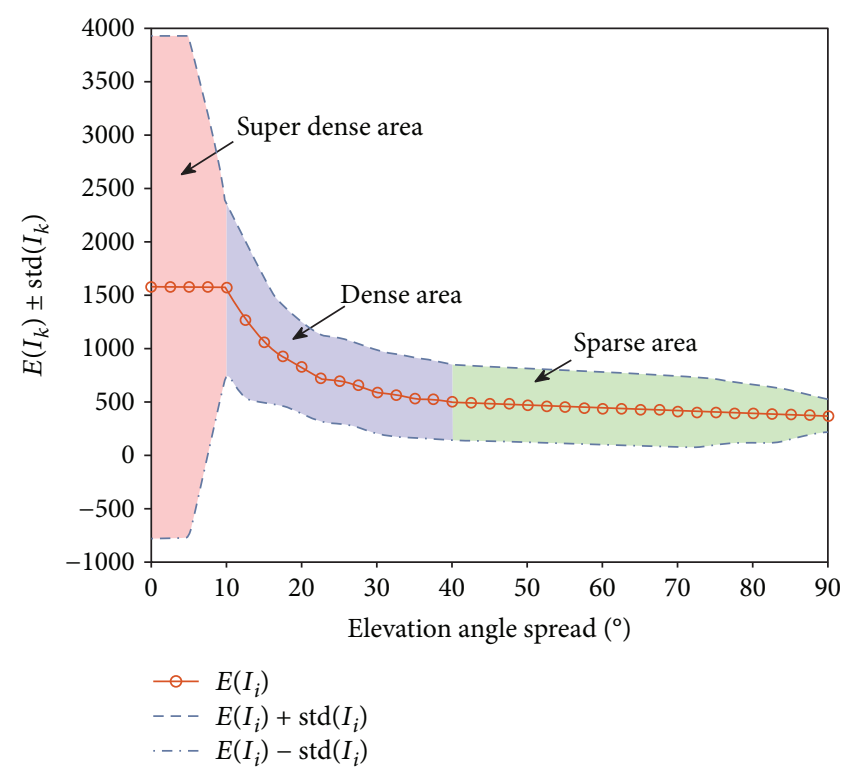

FIgURE 4: Interference variation $E\left[I_{k}\right] \pm \operatorname{std}\left(I_{k}\right)$ against elevation angle spread: $K=5$ and a rectangular antenna array with 100 $(10 \times 10)$ elements at BS with $d_{1}=0.5 \lambda$ and $d_{2}=0.5 \lambda$.

numbers of users or carefully separated users (via scheduling) or both are essential for the satisfactory performance.

4.2. Numerical and Simulation Results: Variance. In Figure 4, we plot the spread of the interference by including levels which are one standard derivation above and below the mean, that is, $\mathbb{E}\left[I_{k}\right] \pm \operatorname{std}\left(I_{k}\right)$, where $\operatorname{std}\left(I_{k}\right)=\sqrt{\operatorname{Var}\left(I_{k}\right)}$. The same parameters such as $K=5$ and a rectangular antenna array with $100(10 \times 10)$ elements at BS with $d_{1}=$ $0.5 \lambda$ and $d_{2}=0.5 \lambda$ are used in the simulation.

While the mean interference level falls with increasing EAS, there remains large variability, especially in the super dense area with small EAS $\left(<10^{\circ}\right)$, where the one standard 
derivation level is approximately 2.5 times greater than the mean interference, which poses a severe challenge for the interference alignment in super dense area. With the EAS increases from $10^{\circ}$ to $40^{\circ}$, the one standard derivation level falls in the same shape as the mean interference does, which indicates a stable interference variance in the dense area. At high EAS in the sparse area, the mean interference keeps a long flat tail and the one standard derivation level gets closer to the mean; the smaller spread of interference would contribute to a more stable system performance. To be noted, the uniform distribution of elevation angle is achieved when the $\mathrm{EAS}=90^{\circ}(\kappa=0)$.

\section{User Scheduling with 3D Angular Separation}

As indicated by the results in Figures 3 and 4 that the interference will decrease rapidly as EAS increases, in this section, we focus on the user scheduling scheme via $3 \mathrm{D}$ angular separation and put forward the user scheduling algorithm to divide multiusers into different independent groups, aiming to maximize the mean angular separation within each group and achieve satisfactory system performance, that is, high sum rate.

5.1. User Scheduling Algorithm with 3D Angular Separation. Assume there are $K$ LoS users in a single cell which are expected to be grouped into $G$ independent groups based on the criteria of maximizing the angular separation of users within each group, which turn out to be a clustering problem. For each LoS user $i$, the location is characterized by $\left(d_{i}, \theta_{i}, \varphi_{i}\right)$, where $d_{i}, \theta_{i}$, and $\varphi_{i}$ denote the distance, the LoS elevation angle, and the LoS azimuth angle from user $i$ to the BS, respectively. The azimuth angle of multiuser, $\varphi$, is assumed as a uniform distribution where $\phi \sim U(0,2 \pi)$, while the elevation angle, $\theta$, follows the typical VM distribution where $\theta \sim V M(\mu, \kappa)$. Multiusers are located evenly between the reference distance for the antenna far-field, $d_{0}$, and the cell radius, $R$. The path loss of the signal from the BS to user $i$ is given by [30, eq (2.28)]

$$
P_{i}=P_{t i} P_{a}\left[\frac{d_{0}}{d_{i}}\right]^{r}, \quad d>d_{0},
$$

where $P_{t i}$ is the signal transmission power to user $i, P_{a}$ is a unitless constant which depends on the antenna characteristics and the average channel attenuation, and $r$ denotes the path loss exponent. Performing the transformation from the spheric coordinate to the Cartesian coordinate, the location vectors of users $i$ and $j$ are given by

$$
\begin{aligned}
& \mathbf{x}_{i}=\left(P_{i} \sin \theta_{i} \cos \phi_{i}, P_{i} \sin \theta_{i} \sin \phi_{i}, P_{i} \cos \theta_{i}\right), \\
& \mathbf{x}_{j}=\left(P_{j} \sin \theta_{j} \cos \phi_{j}, P_{j} \sin \theta_{j} \sin \phi_{j}, P_{j} \cos \theta_{j}\right) .
\end{aligned}
$$

Then, the angular separation between users $i$ and $j, \Delta_{i j}$, is calculated as

$$
\triangle_{i j}=\arccos \left(\mathbf{x}_{i} \cdot \mathbf{x}_{j}^{T}\right)=\frac{\mathbf{x}_{i} \cdot \mathbf{x}_{j}^{T}}{\left|\mathbf{x}_{i}\right|\left|\mathbf{x}_{j}\right|} .
$$

Thus, we could obtain the angular separation matrix $\Delta$ as follows:

$$
\lfloor\Delta\rfloor_{i j}=\Delta_{i j}, \quad(i=1,2, \ldots, K ; j=1,2, \ldots, K),
$$

which will be used as the input of the user scheduling algorithm below:

The following are the parameters: $\Lambda_{k}$ denotes the users selected into the $k$ th group, $I_{G}$ denotes the users which were divided while $I_{L}$ denotes the users left to be grouped, $S_{i}$ denotes the sum of the angular separation between user $i$ and all users in group $G$, and $i_{1}$ and $i_{2}$ denote the users to be grouped in the current step with the target group number $g_{1}$ and $g_{2}$, respectively.

In Algorithm 1, the users in $G$ groups are initialized by steps $4 \sim 14$ where $G$ users with the minimum angular separation among all $K$ users are selected into $G$ different groups, which needs a calculation of $G \times[K(K-1) / 2]$ complexity. Then, the left $K-G$ users are allocated to the existing $G$ groups in steps 15 24 in an order of separating the two nearest users firstly (step 16). Note that, there are $G(G-1) / 2$ cases to allocate users $i_{1}$ and $i_{2}$ to two different groups. For each case, the averaged angular separation of multiuser is calculated. By maximizing the averaged angular separation (step 17), users $i_{1}$ and $i_{2}$ are distributed to groups $\Lambda_{g_{1}}$ and $\Lambda_{g_{2}}$, respectively; thus, the calculation complexity is $(K-G) G(G-1) / 2$. Finally, by performing the iterative suboptimal user scheduling algorithm via 3D angular separation, these $K$ users would be divided into expected $G$ groups and the total calculation complexity is $O\left((G / 2) K^{2}-\right.$ $\left.((G(G-2)) / 2) K-\left(G^{2}(G-1)\right) / 2\right)$, which can be simplified as $O\left((G / 2) K^{2}\right)$ as $G \ll K$ usually.

In the following part of this section, the system sum rate will be calculated; then, the comparative results and analysis will be presented under different expected group numbers, that is, $G=1,2,3,4,5$. To be noted, when $G=1$, all users are in the same group and no user scheduling is performed. When $G>1$, as a reference, we present the results of the system sum rate for user random scheduling as well, which means $K$ users are randomly allocated to $G$ groups without selection.

5.2. System Sum Rate Calculation. Based on Algorithm 1, users will be divided into different groups with the largest angular separation to avoid the potential interference. As users in different groups will be served in orthogonal resource blocks, the mutual interference between users in different groups is assumed 0; thus, the multiuser sum rate can be calculated as

$$
\bar{R}=\sum_{g=1}^{G} \sum_{k \in \Lambda_{g}} \log _{2}\left(1+\frac{M N \beta_{k}}{\left(\beta_{k} / M N\right) I_{k}^{(g)}+\sigma_{z}^{2}}\right),
$$

where $I_{k}^{(g)}$ denotes the interference to user $k$ from other users in group $\Lambda_{g}$. 


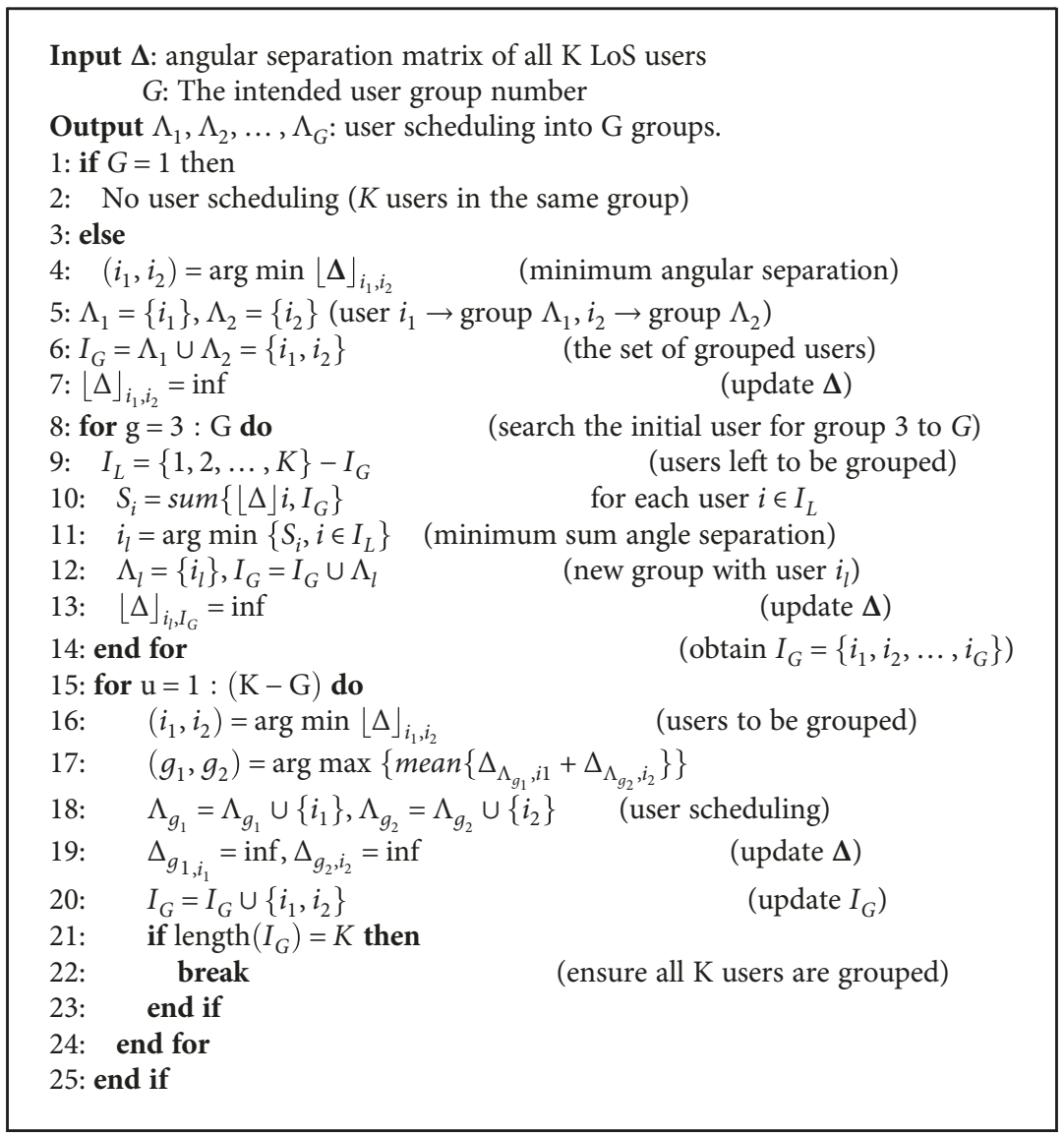

Algorithm 1: User scheduling with 3D angular separation.

We assume that the angles of all users are independent; the first moment $\mathbb{E}\left[I_{k}\right]$ in (9) can be simplified as

$$
\mathbb{E}\left[I_{k}\right]=\sum_{j \neq k}^{K} \mathbb{E}\left[I_{k j}\right]=(K-1) \mathbb{E}\left[I_{k j}\right] .
$$

Thus, we would obtain

$$
\mathbb{E}\left[I_{k}^{(g)}\right]=\sum_{j \neq k, j \in \Lambda_{g}}^{L^{(g)}} \mathbb{E}\left[I_{k j}\right]=\left(L^{(g)}-1\right) \mathbb{E}\left[I_{k j}\right]
$$

5.3. Simulative Results and Analysis. In the simulation, we assume the same antenna array, the URA in Figure 1 with 100 elements $(10 \times 10)$ and half wavelength antenna spacing in both elevation and azimuth domains $\left(d_{1}=0.5\right.$ and $d_{2}=0.5$ ), is used at BS. $K=20$ users are distributed evenly around the BS in the azimuth domain while the elevation angles obey a von Mises distribution in the LoS MIMO system. The comparative results of the system sum rate between user scheduling in Algorithm 1 and random scheduling without user selection are presented in Figure 5 under different EASs, that is, EAS $=10^{\circ}, 30^{\circ}, 50^{\circ}$, which would provide insights on the effects of user scheduling via $3 \mathrm{D}$ angular separation on the system performance.
From the results in Figure 5, an obvious feature is that a large sum rate gain is obtained by performing the user scheduling via 3D angular separation (solid red curves) as compared to the sum rate of no scheduling (solid red curve with $G=1$ ) and random scheduling (dotted dark curves). Furthermore, the more groups the multiusers are expected to be divided into, the higher the sum rate is observed, and this sum rate gain will be further enhanced. Taking the results in Figure 5(a) as an example, when the expected group number $G$ increases from 1 to $2,3,4$, and 5 , the $0.5 \mathrm{CDF}$ point sum rate gains harvested by user scheduling scheme are $3.9,7.3,10.3$, and $13.3 \mathrm{bps} / \mathrm{Hz}$, respectively, under $\mathrm{EAS}=10^{\circ}$. Once the multiusers are scheduling into different independent groups, the intergroup interference would be eliminated, which contributes to a lower total system interference and higher sum rate. Meanwhile, by performing the user scheduling algorithm with maximal angular separation among users within each group, the intragroup interference will be lowered; thus, the system sum rate is further improved.

Increasing the EAS from $10^{\circ}$ to $30^{\circ}$ and $50^{\circ}$ where users are better separated in the $3 \mathrm{D}$ space, the corresponding results of the system sum rate are illustrated in Figures 5(b) and 5(c), respectively. As compared to the results in Figure 5(a), the system sum rate is improved by larger EAS as expected. A more specific result of the sum rate gain 


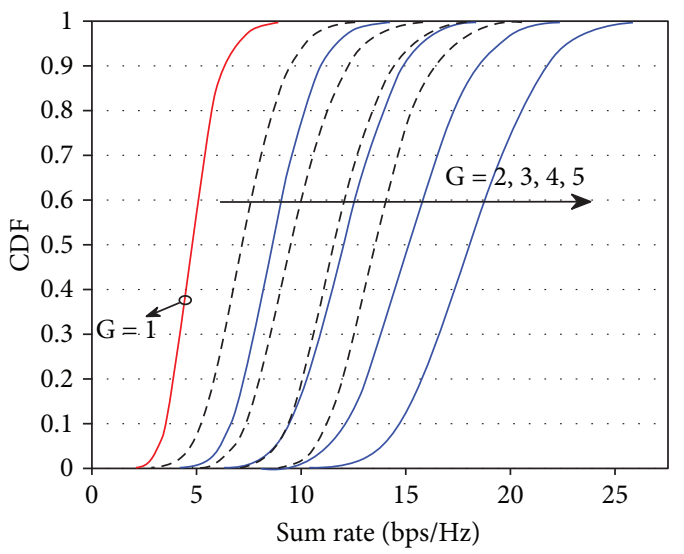

$$
\begin{aligned}
& \text { - No scheduling } \\
& \text {--- Random scheduling } \\
& \text { - User scheduling }
\end{aligned}
$$

(a)

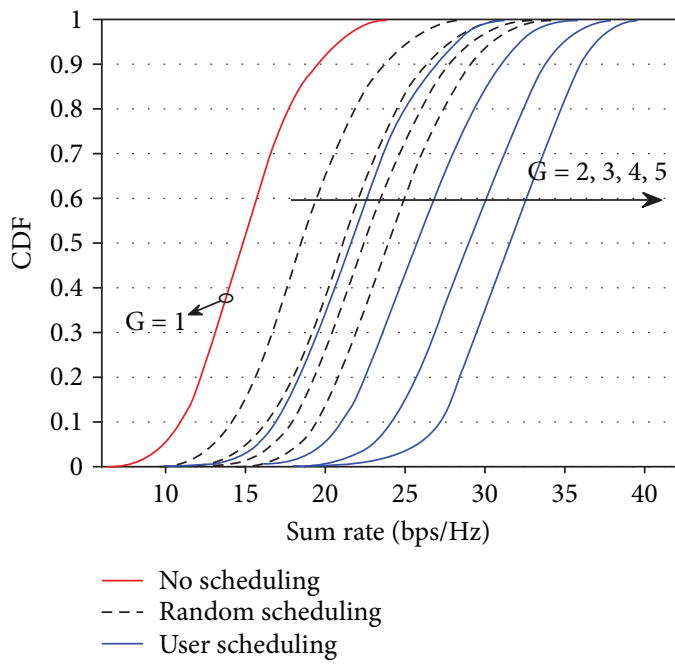

(b)

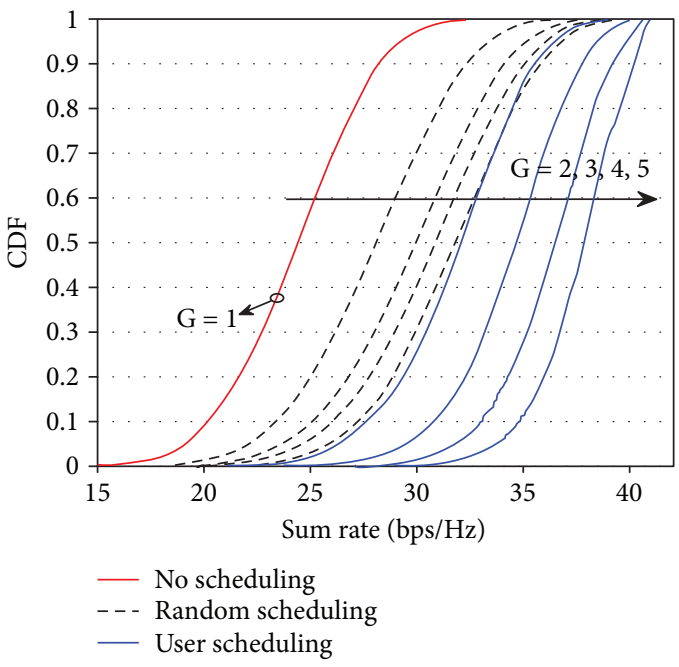

(c)

FiguRe 5: Multiuser sum rate under different user group values. (a) Multiuser sum rate under different user group values: $K=20$, $\Phi \sim U(0,2 \pi)$, and $\theta \sim V M(0,8.52)$ with $\mathrm{EAS}=10^{\circ}$. (b) Multiuser sum rate under different user group values: $K=20, \Phi \sim U(0,2 \pi)$, and $\theta \sim \operatorname{VM}(0,1.48)$ with EAS $=30^{\circ}$. (c) Multiuser sum rate under different user group values: $K=20, \Phi \sim U(0,2 \pi)$, and $\theta \sim V M(0,0.14)$ with $\mathrm{EAS}=50^{\circ}$.

TABLE 1: The sum rate gain and gain ratio $(\mathrm{CDF}=0.5)$ harvested by user scheduling with $3 \mathrm{D}$ angular separation when $G=5$ where $L^{(g)}$ denotes the user number in group $\Lambda_{g}$ while the moment of interference, $\mathbb{E}\left[I_{k j}\right]$, can be found in (B.3) in the Appendix B.

\begin{tabular}{llcr}
\hline User scheduling $(G=5)$ versus & & No scheduling $(G=1)$ & Random scheduling $(G=5)$ \\
\hline & EAS $=10^{\circ}$ & $14.0 \mathrm{bps} / \mathrm{Hz} / 345 \%$ & $4.6 \mathrm{bps} / \mathrm{Hz} / 34 \%$ \\
Sum rate gain $(\mathrm{bps} / \mathrm{Hz}) /$ gain ratio & $\mathrm{EAS}=30^{\circ}$ & $16.9 \mathrm{bps} / \mathrm{Hz} / 114 \%$ & $7.7 \mathrm{bps} / \mathrm{Hz} / 32 \%$ \\
& $\mathrm{EAS}=50^{\circ}$ & $13.4 \mathrm{bps} / \mathrm{Hz} / 55 \%$ & $6.1 \mathrm{bps} / \mathrm{Hz} / 19 \%$ \\
\hline
\end{tabular}

against EAS is presented in Table 1. As we can see, a large sum rate gain is obtained by user scheduling with $3 \mathrm{D}$ angular separation, and the gain ratio decreases as the EAS increases. For small EAS $\left(10^{\circ}\right)$, the sum rate gain can even be 3.5 times larger than that of no scheduling case, which highlights the importance of user scheduling for interference alignment in super dense area.
Although the above observations are for multiuser interference in a single cell, similar results of significant sum rate gain due to the proposed user scheduling algorithm can be expected for multiple cells in the future work. As we can conclude, in environments with considerable angular diversity, user scheduling based on angular separations will be an effective tool in interference alignment. 


\section{Conclusion}

In this article, we derive the exact moments of interference in 3D LoS downlink channel where the multiusers are assumed as a uniform distribution in the azimuth domain and von Mises distribution in the elevation counterpart. The results demonstrate that the mean interference decreases as the antenna number decreases, as the antenna spacing increases and the angular separation increases. For small EAS, a large interference is observed and the spread of interference is massive; thus, carefully separated users via scheduling are essential for the satisfactory performance. Based on the 3D angular separation, we put forward a user scheduling algorithm and group users into independent subspaces with corresponding orthogonal fraction of resource blocks. Simulative results show that the system performance is improved in terms of sum rate as compared to the no user scheduling and random scheduling cases, that is, by grouping 20 users into 5 subspaces, the average sum rate increases from $4.1 \mathrm{bps} / \mathrm{Hz}$ (no scheduling) and $13.5 \mathrm{bps} / \mathrm{Hz}$ (random scheduling) to $18.1 \mathrm{bps} / \mathrm{Hz}$ (user scheduling) at $\mathrm{E}$ $\mathrm{AS}=10^{\circ}$, where the sum rate gain ratio can be as high as $334 \%$ and $34 \%$, respectively.

Collectively, the LoS interference statistics provide insights into the effects of antenna number at BS, antenna spacing, and multiuser angular separation on the system performance. The large sum rate gain and gain ratio harvested by user scheduling highlight the importance of $3 \mathrm{D}$ angular separation for interference alignment, especially for scenarios with multiuser in super dense area. As we can conclude, the user scheduling algorithm based on $3 \mathrm{D}$ angular separation enables us a promising way in interference control and management.

\section{Appendix}

\section{A. Proof of Lemma 1}

Given the uniform distribution in azimuth domain with $\phi \sim \mathcal{U}[0,2 \pi]$, we have

$$
\begin{aligned}
& \mathbb{E}\left[e^{-j(m-p) u_{k}} e^{-j(n-q) v_{k}}\right] \\
& \quad=\int_{\theta} e^{-j(m-p) u_{k}}\left(\int_{\phi} e^{-j(n-q)(2 \pi / \lambda) d_{2} \sin \theta \cos \phi} \frac{1}{2 \pi} d \phi\right) f(\theta) d \theta \\
& =\int_{\theta} e^{-j(m-p)(2 \pi / \lambda) d_{1} \cos \theta} J_{0}\left((n-q) \frac{2 \pi}{\lambda} d_{2} \sin \theta\right) f(\theta) d \theta \\
& =\int_{\theta} e^{-j 2 \pi d_{z}^{m p} \cos \theta} J_{o}\left(2 \pi d_{y}^{n q} \sin \theta\right) f(\theta) d \theta
\end{aligned}
$$

For simplicity, we ignore the subscript $k$ in the derivation. Then, substituting $f(\theta)$ in (14) with $\theta \sim V M(\kappa, \pi / 2)$ and the approximation (15) into (A.1), we would obtain

$$
\begin{aligned}
& \mathbb{E}\left[e^{-j(m-p) u_{k}} e^{-j(n-q) v_{k}}\right] \\
& \quad=\int_{0}^{\pi} \frac{e^{\kappa \sin \theta}}{2 \pi I_{0}(\kappa)} J_{0}\left(2 \pi d_{y}^{n, q} \sin \theta\right) e^{-j 2 \pi d_{z}^{m, p} \cos \theta} d \theta \\
& \quad \approx \int_{0}^{\pi} \frac{\left(e^{\kappa}-1\right) \sin \theta^{L}+1}{2 \pi I_{0}(\kappa)} J_{0}\left(2 \pi d_{y}^{n, q} \sin \theta\right) e^{-j 2 \pi d_{z}^{m, p} \cos \theta} d \theta \\
& \quad=\int_{0}^{\pi}\left(a+b \sin \theta^{L}\right) J_{0}\left(2 \pi d_{y}^{n, d} \sin \theta\right) e^{-j 2 \pi d_{z}^{m, p} \cos \theta} d \theta,
\end{aligned}
$$

where $a$ and $b$ are defined in (24).

Further, expanding

$$
e^{-j 2 \pi d_{z}^{m, p} \cos \theta_{k}}=J_{0}\left(-2 \pi d_{z}^{m, p}\right)+2 \sum_{r=1}^{R} j^{r} J_{r}\left(-2 \pi d_{z}^{m, p}\right) \cos (r \theta),
$$

and substituting (A.3) into (A.2), we would obtain (A.4). Then, the integral calculation of (A.4) based on lemmas in $[29$, eq. $(6.6818,6.6819)]$ would lead to (A.5), which can be further simplified as (A.6) with the desired parameters $A$ and $B$ in (17) and (18), respectively. Thus, the remaining work in the proof of Lemma 1 will be the derivation of the exact expressions of $C$ and $D$ in (19)-(22) based on the preliminary definitions below:

$$
\begin{aligned}
& \mathbb{E}\left[e^{-j(m-p) u_{k}} e^{-j(n-q) v_{k}}\right] \\
& \approx J_{0}\left(2 \pi d_{z}^{m, p}\right) \int_{0}^{\pi}\left(a+b \sin \theta^{n}\right) J_{0}\left(2 \pi d_{y}^{n, q} \sin \theta\right) d \theta \\
&+2 \sum_{r=1}^{R} j^{r} J_{r}\left(-2 \pi d_{z}^{m, p}\right) \int_{0}^{\pi}\left(a+b \sin \theta^{n}\right) J_{0} \\
& \cdot\left(2 \pi d_{y}^{n, q} \sin \theta\right) \cos (r \theta) d \theta \\
& \mathbb{E}\left[e^{-j(m-p) u_{k}} e^{-j(n-q) v_{k}}\right] \\
&=a \pi J_{0}\left(-2 \pi d_{z}^{m, p}\right) J_{0}^{2}\left(\pi d_{y}^{n, q}\right) \\
& \quad+2 a \sum_{r=1}^{R} j^{r} J_{r}\left(-2 \pi d_{z}^{m, p}\right) \pi \cos \frac{r}{2} \pi J_{-(r / 2)}\left(\pi d_{y}^{n, q}\right) J_{r / 2}\left(\pi d_{y}^{n, q}\right) \\
&+b J_{0}\left(2 \pi d_{z}^{m, p}\right) \int_{0}^{\pi} \sin \theta^{n} J_{0}\left(2 \pi d_{y}^{n, q} \sin \theta\right) d \theta \\
& \quad+2 b \sum_{r=1}^{R} j^{r} J_{r}\left(-2 \pi d_{z}^{m, p}\right) \int_{0}^{\pi} \sin \theta^{n} J_{0} \\
& \quad\left(2 \pi d_{y}^{n, q} \sin \theta\right) \cos (r \theta) d \theta
\end{aligned}
$$

$$
\begin{aligned}
\mathbb{E}\left[e^{-j(m-p) u_{k}} e^{-j(n-q) v_{k}}\right]= & A+B+b J_{0}\left(2 \pi d_{z}^{m, p}\right) C \\
& +2 b \sum_{r=1}^{R} j^{r} J_{r}\left(-2 \pi d_{z}^{m, p}\right) D
\end{aligned}
$$




$$
\begin{aligned}
& C=\int_{0}^{\pi} \sin \theta^{L} J_{0}\left(2 \pi d_{y}^{n, q} \sin \theta\right) d \theta, \\
& D=\int_{0}^{\pi} \sin \theta^{L} J_{0}\left(2 \pi d_{y}^{n, q} \sin \theta\right) \cos (r \theta) d \theta .
\end{aligned}
$$

Case 1. When $L=2 L_{0}$ is even, substituting (26) into (A.7) and (A.8) will lead to the result in (A.9) and (A.10), and then performing the integral calculation based on [29, eq. (6.6818, $6.6819)$ ] will lead to the desired result in (19) and (20).

$$
\begin{aligned}
C & =\int_{0}^{\pi} \sum_{l=0}^{L_{0}} k_{l} \cos 2 l \theta J_{0}\left(2 \pi d_{y}^{n, q} \sin \theta\right) d \theta \\
D & =\int_{0}^{\pi} \sum_{l=0}^{L_{0}} k_{l} \cos 2 l \theta \cos (r \theta) J_{0}\left(2 \pi d_{y}^{n, q} \sin \theta_{k}\right) d \theta_{k} \\
& =\sum_{l=0}^{L_{0}} k_{l} \int_{0}^{\pi} \frac{\cos \left(2 l_{1}\right) \theta+\cos \left(2 l_{2}\right) \theta}{2} J_{0}\left(2 \pi d_{y}^{n, q} \sin \theta\right) d \theta
\end{aligned}
$$

Case 2. When $L=2 L_{1}-1$ is odd, substituting (28) into (A.7) and (A.8) will lead to the result in (A.11) and (A.12), respectively, and then performing the integral calculation based on $[29$, eq. $(6.6818,6.6819)]$ will lead to the desired result in (21) and (22).

$$
\begin{aligned}
C & =\int_{0}^{\pi} \sum_{l=1}^{n_{1}} k_{l} \sin (2 l-1) \theta J_{0}\left(2 \pi d_{y}^{n, p} \sin \theta\right) d \theta \\
D & =\int_{0}^{\pi} \sum_{l=1}^{n_{1}} k_{l} \sin (2 l-1) \theta \cos (r \theta) J_{0}\left(2 \pi d_{y}^{n, q} \sin \theta\right) d \theta \\
& =\sum_{l=1}^{L_{1}} k_{l} \int_{0}^{\pi} \frac{\sin \left(2 l_{3}\right)+\sin \left(2 l_{4}\right) \theta}{2} J_{0}\left(2 \pi d_{y}^{n, q} \sin \theta\right) d \theta
\end{aligned}
$$

\section{B. Proof of Theorem 1}

As we can see from (8), we need to obtain the term $\mathbf{a}_{k} \mathbf{a}_{j}^{+}$based on the definition in (4).

$$
\begin{aligned}
\mathbf{a}_{k} \mathbf{a}_{j}^{+}= & {\left[\mathbf{H}_{h, k}, \mathbf{H}_{h, k} e^{-j u_{k}}, \ldots, \mathbf{H}_{h, k} e^{-j(M-1) u_{k}}\right] } \\
& \cdot\left[\mathbf{H}_{h, j}^{*}, \mathbf{H}_{h, j}^{*} e^{j u_{j}}, \ldots, \mathbf{H}_{h, k}^{*} e^{j(M-1) u_{j}}\right]^{T} \\
= & \mathbf{H}_{h, k} \mathbf{H}_{h, j}^{*}\left(1+e^{-j\left(u_{k}-u_{j}\right)}+\cdots+e^{-j(M-1)\left(u_{k}-u_{j}\right)}\right) \\
= & \sum_{n=0}^{N-1} e^{-j n\left(v_{k}-v_{j}\right)} \sum_{m=0}^{M-1} e^{-j m\left(u_{k}-u_{j}\right)} \\
= & \sum_{m=0}^{M-1} \sum_{n=0}^{N-1} e^{-j m\left(u_{k}-u_{j}\right)} e^{-j n\left(v_{k}-v_{j}\right)} .
\end{aligned}
$$

Thus, substituting (B.1) into (8) will give

$$
\begin{aligned}
\mathbb{E}\left[I_{k j}\right] & =\mathbb{E}\left[\sum_{m=0}^{M-1} \sum_{n=0}^{N-1} e^{-j m\left(u_{k}-u_{j}\right)} e^{-j n\left(v_{k}-v_{j}\right)} \sum_{p=0}^{M-1} \sum_{q=0}^{N-1} e^{j p\left(u_{k}-u_{j}\right)} e^{j q\left(v_{k}-v_{j}\right)}\right] \\
& =\mathbb{E}\left[\sum_{m=0}^{M-1} \sum_{n=0}^{N-1} \sum_{p=0}^{M-1} \sum_{q=0}^{N-1} e^{-j(m-p)\left(u_{k}-u_{j}\right)} e^{-j(n-q)\left(v_{k}-v_{j}\right)}\right] \\
& =\mathbb{E}\left[\sum_{m, n, p, q} e^{-j(m-p) u_{k}} e^{-j(n-q) v_{k}} e^{j(m-p) u_{j}} e^{j(n-q) v_{j}}\right],
\end{aligned}
$$

where $\Sigma_{m, n, p, q}$ denotes $\Sigma_{m=0}^{M-1} \sum_{m=0}^{N-1} \sum_{p=0}^{M-1} \sum_{q=0}^{N-1}$.

As users $k$ and $j$ are assumed to be independent, then (B.2) can be further simplified as

$$
\begin{aligned}
\mathbb{E}\left|I_{k j}\right| & =\sum_{m, n, p, q} \mathbb{E}\left[e^{-j(m-p) u_{k}} e^{-j(n-q) v_{k}}\right] \mathbb{E}\left[e^{j(m-p) u_{j}} e^{j(n-q) v_{j}}\right] \\
& =\sum_{m, n, p, q}\left|\mathbb{E}\left[e^{-j(m-p) u_{k}} e^{-j(n-q) v_{k}}\right]\right|^{2}
\end{aligned}
$$

Substituting $\mathbb{E}\left[I_{k j}\right]$ of (B.3) into (38), we can obtain

$$
\mathbb{E}\left[I_{k}\right]=(K-1) \mathbb{E}\left[e^{-j(m-p) u_{k}} e^{-j(n-q) v_{k}}\right] .
$$

Using Lemma 1 for the expectation expressions gives the desired result of (30).

\section{Proof of Theorem 2}

Assuming the angle distributions of different users are independent, then from (9), we have

$$
\begin{aligned}
\mathbb{E}\left[I_{k}^{2}\right] & =\mathbb{E}\left[\left(\sum_{j \neq k}^{K} I_{j k}\right)^{2}\right]=\mathbb{E}\left[\sum_{j \neq k}^{K} \sum_{i \neq k}^{K} I_{j k} I_{i k}\right] \\
& =\sum_{j \neq k}^{K} \sum_{i \neq k}^{K} \mathbb{E}\left[I_{k j} I_{k i}\right]=(K-1)^{2} \mathbb{E}\left[I_{k j} I_{k i}\right] .
\end{aligned}
$$

Define $d_{y}^{n_{1} n_{2}, q_{1} q_{2}}$ and $d_{y}^{m_{1} m_{2}, p_{1} p_{2}}$ as

$$
\begin{aligned}
d_{y}^{n_{1} n_{2}, q_{1} q_{2}} & =\frac{\left(n_{1}+n_{2}-q_{1}-q_{2}\right) d_{2}}{\lambda}, \\
d_{z}^{m_{1} m_{2}, p_{1} p_{2}} & =\frac{\left(m_{1}+m_{2}-p_{1}-p_{2}\right) d_{1}}{\lambda} .
\end{aligned}
$$

Similar as (B.3), we can have the following result after several simplifications

$$
\begin{aligned}
\mathbb{E}\left[I_{k j} I_{k i}\right]= & \sum_{p_{1} q_{1} m_{1} n_{1} p_{2} q_{2} m_{2} n_{2}} \mathbb{E}\left[e^{-j 2 \pi d_{z}^{m_{1} m_{2}, p 1 p 2} u_{k}} e^{-j 2 \pi d_{y}^{n_{1} n_{2}, q_{1} q_{2}} v_{k}}\right] \mathbb{E} \\
& \cdot\left[e^{j 2 \pi d_{z}^{m_{1}, p 1}} u_{j} e^{j 2 \pi d_{y}^{n_{1}, q_{1}}} v_{j}\right] \mathbb{E}\left[e^{j 2 \pi d_{z}^{m_{2}, p_{2}} u_{i}} e^{j 2 \pi d_{y}^{n_{2}, q_{2}} v_{i}}\right] .
\end{aligned}
$$

Using Lemma 1 for the expectation expressions gives the desired result of (31). 


\section{Conflicts of Interest}

The authors declare that they have no conflicts of interest.

\section{Acknowledgments}

The authors would like to thank Professor Peter J. Smith sincerely for his guidance and supervision of this article. He helped a lot in the proof of Lemma 1 and the main idea of the user scheduling algorithm. The authors would like to thank Dr. Pawel A. Dmochowski very much for his suggestions in discussing the work on LoS interference moments. The research is supported in part by the National Natural Science Foundation of China with the project name Theoretical Modeling and Experiment Research of Propagation Channel with no. 61322110, in part by the Ministry of Education-China Mobile Research Fund-with the project name "5G Wireless Transmission Key Technology Research and Evaluation for 3GPP Standardization" with MCM20160105, and in part by the National Science and Technology Major Program of the Ministry of Science and Technology, with the project name Research on 5G channel emulation and performance validation with 2018ZX03001031.

\section{References}

[1] E. Larsson, O. Edfors, F. Tufvesson, and T. Marzetta, "Massive MIMO for next generation wireless systems," IEEE Communications Magazine, vol. 52, no. 2, pp. 186-195, 2014.

[2] B. Li, D. Zhu, and P. Liang, "Small cell in-band wireless backhaul in massive MIMO systems: a cooperation of nextgeneration techniques," IEEE Transactions on Wireless Communications, vol. 14, no. 12, pp. 7057-7069, 2015.

[3] T. S. Rappaport, Shu Sun, R. Mayzus et al., "Millimeter wave mobile communications for 5G cellular: it will work!," IEEE Access, vol. 1, pp. 335-349, 2013.

[4] Y.-H. Cho and J.-J. Kim, "Line-of-sight MIMO channel in millimeterwave beamforming system: modeling and prototype results," in The 81st IEEE Vehicular Technology Conference (VTC Spring), pp. 1-5, Glasgow, UK, May 2015.

[5] C. Feng, Y. Jing, and S. Jin, "Interference and outage probability analysis for massive MIMO downlink with MF precoding," IEEE Signal Processing Letters, vol. 23, no. 3, pp. 366-370, 2016.

[6] J. Robler and J. Huber, "Matched filter for transmission over channels with ISI employing the distribution of interference," in The 57th IEEE Semiannual Vehicular Technology Conference (VTC Spring), vol. 4, pp. 2648-2652, Jeju, South Korea, April 2003.

[7] S. Karmakar and M. K. Varanasi, "The capacity region of the MIMO interference channel and its reciprocity to within a constant gap," IEEE Transactions on Information Theory, vol. 59, no. 8, pp. 4781-4797, 2013.

[8] N. Zhao, F. R. Yu, M. Jin, Q. Yan, and V. C. M. Leung, "Interference alignment and its applications: a survey, research issues, and challenges," IEEE Communications Surveys \& Tutorials, vol. 18, no. 3, pp. 1779-1803, 2016.

[9] H. Q. Ngo, E. G. Larsson, and T. L. Marzetta, "Aspects of favorable propagation in massive MIMO," in the 22nd IEEE
Signal Processing Conference (EUSIPCO), pp. 76-80, Lisbon, Portugal, September 2014.

[10] J. Chen, "When does asymptotic orthogonality exist for very large arrays," in 2013 IEEE Global Communications Conference (GLOBECOM), pp. 4146-4150, Atlanta, GA, USA, December 2013.

[11] T. Halsig and B. Lankl, "Array size reduction for high-rank LOS MIMO ULAs," IEEE Wireless Communications Letters, vol. 4, no. 6, pp. 649-652, 2015.

[12] X. Pu, K. L. Law, S. Shao, and Y. Tang, "On the capacity for 3D LoS MIMO channels in short-range millimeter wave communications," in 11th International Conference on Wireless Communications, Networking and Mobile Computing (WiCOM 2015), pp. 1-6, Shanghai, China, September 2015.

[13] P. Chandhar, D. Danev, and E. G. Larsson, "On ergodic rates and optimal array geometry in line-of-sight massive MIMO," in 2016 IEEE 17th International Workshop on Signal Processing Advances in Wireless Communications (SPAWC), pp. 16, Edinburgh, UK, July 2016.

[14] W. Tan, S. Jin, J. Wang, and Y. Huang, "Achievable sum-rate analysis for massive MIMO systems with different array configurations," in 2015 IEEE Wireless Communications and Networking Conference (WCNC), pp. 316-321, New Orleans, LA, USA, March 2015.

[15] Y. Hu, Y. Hong, and J. Evans, "Angle-of-arrival-dependent interference modeling in Rician massive MIMO," IEEE Transactions on Vehicular Technology, vol. 66, no. 7, pp. 6171-6183, 2017.

[16] Y. Hu, Y. Hong, and J. S. Evans, "Interference in LoS massive MIMO is well approximated by a beta-mixture," in 2015 IEEE International Conference on Communication Workshop (ICCW), pp. 1137-1142, London, UK, June 2015.

[17] A. Iyer, C. Rosenberg, and A. Karnik, "What is the right model for wireless channel interference," IEEE Transactions on Wireless Communications, vol. 8, no. 5, pp. 2662-2671, 2009.

[18] S. Jin, W. Tan, M. Matthaiou, J. Wang, and K.-K. Wong, "Statistical eigenmode transmission for the MU-MIMO downlink in Rician fading," IEEE Transactions on Wireless Communications, vol. 14, no. 12, pp. 6650-6663, 2015.

[19] I. Santamaria, O. Gonzalez, W. R. H. Jr., and S. W. Peters, "Maximum sum-rate interference alignment algorithms for MIMO channels," in 2010 IEEE Global Telecommunications Conference GLOBECOM, pp. 1-6, Miami, FL, USA, December 2010.

[20] L. Ruan, V. K. N. Lau, and M. Z. Win, "Generalized interference alignment-part I: theoretical framework," IEEE Transactions on Signal Processing, vol. 64, no. 10, pp. 2675-2687, 2016.

[21] E. Castaneda, A. Silva, A. Gameiro, and M. Kountouris, “An overview on resource allocation techniques for multi-user MIMO systems," IEEE Communications Surveys \& Tutorials, vol. 19, no. 1, pp. 239-284, 2017.

[22] S. H. Chae and S.-Y. Chung, "Blind interference alignment for a class of k-user line-of-sight interference channels," IEEE Transactions on Communications, vol. 60 , no. 5, pp. 11771181, 2012.

[23] L. H. Grokop, D. N. C. Tse, and R. D. Yates, "Interference alignment for line-of-sight channels," IEEE Transactions on Information Theory, vol. 57, no. 9, pp. 5820-5839, 2011. 
[24] A. Bayesteh and A. K. Khandani, "On the user selection for MIMO broadcast channels," IEEE Transactions on Information Theory, vol. 54, no. 3, pp. 1086-1107, 2008.

[25] S. A. Jafar, "Interference alignment-a new look at signal dimensions in a communication network," Foundations and Trends® in Communications and Information Theory, vol. 7, no. 1, pp. 1-134, 2010.

[26] Y. Yu, J. Zhang, P. J. Smith, and P. A. Dmochowski, “Theoretical analysis of 3-D channel spatial correlation and capacity," IEEE Communications Letters, vol. 22, no. 2, pp. 420-423, 2018.

[27] D. Ying, F. W. Vook, T. A. Thomas, D. J. Love, and A. Ghosh, "Kronecker product correlation model and limited feedback codebook design in a 3d channel model," in 2014 IEEE International Conference on Communications (ICC), pp. 58655870, Sydney, NSW, Australia, June 2014.

[28] W. Tan, P. J. Smith, H. A. Suraweera, M. Matthaiou, and S. Jin, "Spectral efficiency of multi-user mmWave systems with uniform linear arrays and MRT," in 2016 IEEE 83rd Vehicular Technology Conference (VTC Spring), pp. 1-5, Nanjing, China, May 2016.

[29] I. S. Gradshteyn and I. M. Ryzhik, Table of Integrals, Series, and Products, Academic Press, 7th edition, 2014.

[30] A. J. Goldsmith, Wireless communications, Cambridge University Press, 2004. 


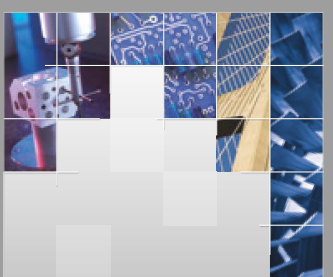

\section{Enfincering}
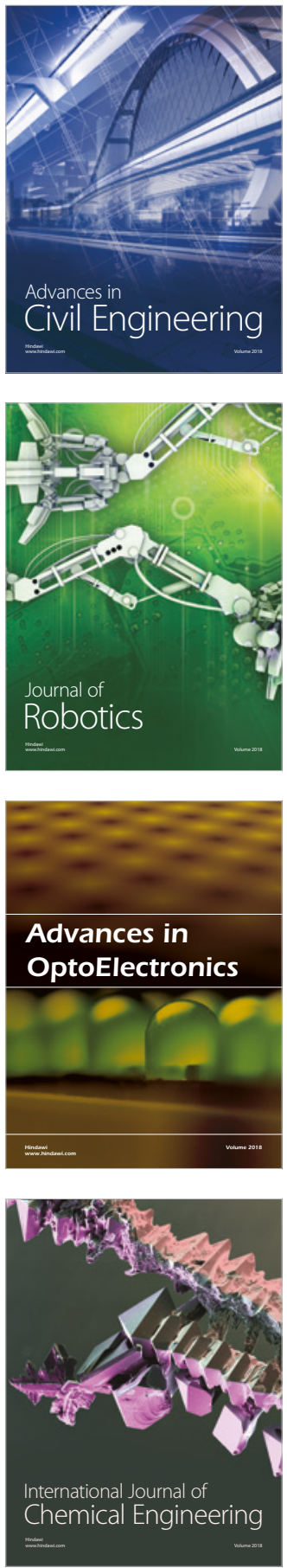

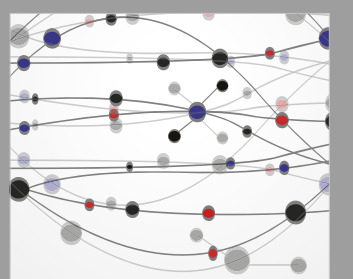

\section{Rotating \\ Machinery}

The Scientific World Journal

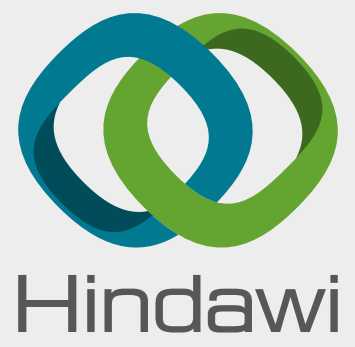

Submit your manuscripts at

www.hindawi.com
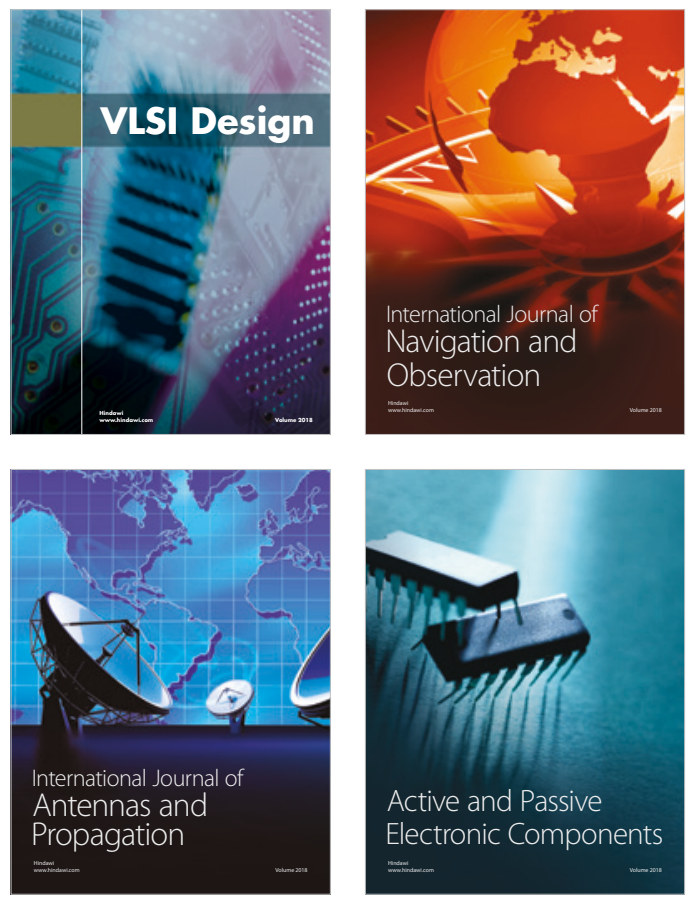
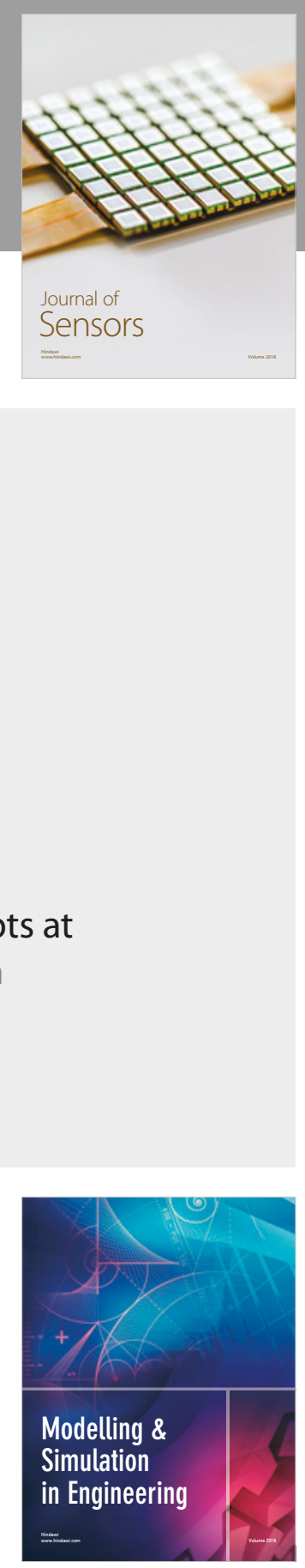

\section{Advances \\ Multimedia}
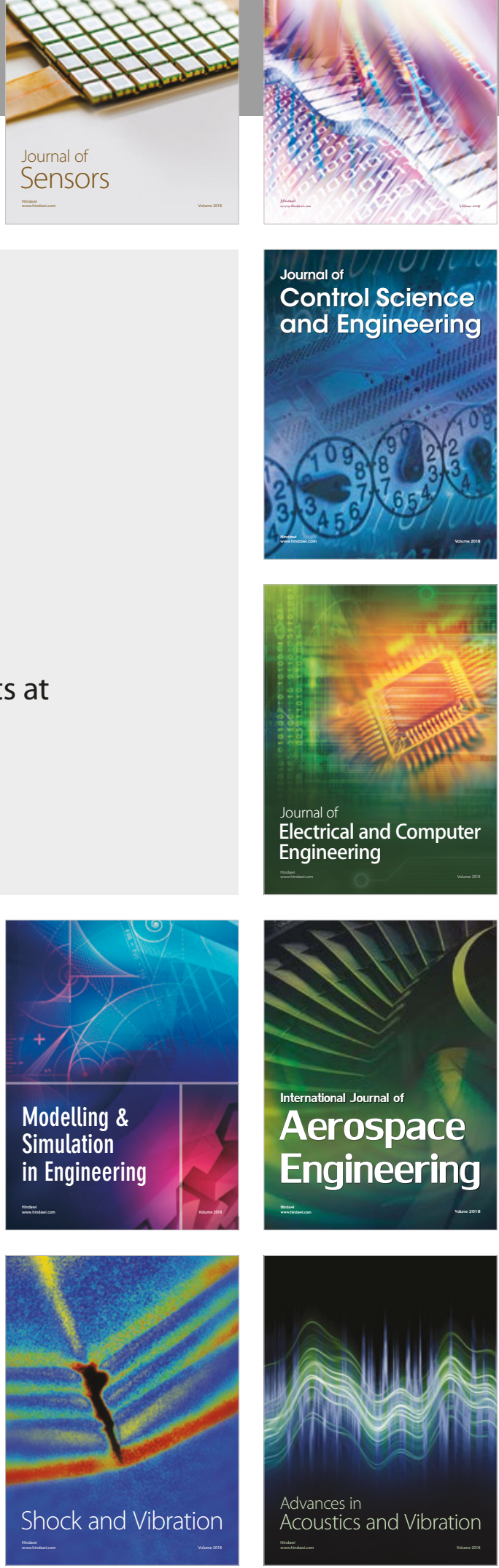